Annuaire suisse de politique de développement

12 | 1993

Annuaire Suisse - Tiers Monde 1993

\title{
VII. Chronologie Suisse et internationale
}

\section{(2) OpenEdition}

1 Journals

Édition électronique

URL : http://journals.openedition.org/aspd/1412

DOI : $10.4000 /$ aspd. 1412

ISSN : 1663-9669

Éditeur

Institut de hautes études internationales et du développement

\section{Édition imprimée}

Date de publication : 1 février 1993

Pagination : 164-170

ISSN : 1660-5934

\section{Référence électronique}

«VII. Chronologie Suisse et internationale », Annuaire suisse de politique de développement [En ligne],

12 | 1993, mis en ligne le 30 avril 2013, consulté le 08 septembre 2020. URL : http://

journals.openedition.org/aspd/1412; DOI : https://doi.org/10.4000/aspd.1412 


\section{Chronologie Suisse et internationale}

\section{Septembre 1991}

\section{3-19 septembre}

Conférence sur le Programme d'action des Nations-Unies pour le redressement économique de l'Afrique, dans le cadre de l'Assemblée 4 septembre générale des Nations-Unies.

La Suisse accorde un crédit mixte de 60 millions de francs à l'Egypte (constitué pour moitié par un don de la Confédération et pour l'autre 5 septembre moitié par des crédits bancaires).

Conférence annuelle de la DDA sur le thème de la recherche et le 6-13 septembre transfert de technologie dans la coopération au développement.

17 septembre

Session du Conseil international du cacao, Londres.

Le Conseil National approuve l'adhésion de la Suisse au FMI et à la 18 septembre

Banque Mondiale.

Le Conseil National approuve l'adhésion de la Suisse aux deux pactes des Nations-Unies sur les droits de l'homme de 1966. Le pacte I contient un catalogue des droits économiques, sociaux et culturels. Le pacte II 19-20 septembre

garanti des droits fondamentaux et la liberté.

Symposium d'automne organisé par Swissaid sur le thème "Des plantes 23-27 septembre

pour les hommes ou des brevets pour la bio-industrie?", Berne.

Réunion du Conseil international du café, Londres. 23 septembre- 4 octobre

CNUCED, première partie de la 38ème session du Conseil du commerce et du développement, Genève. 


\section{7 septembre}

Le Conseil Fédéral présente le Message sur la continuation de la coopération avec les pays de l'Est et de l'Europe centrale pour un montant de $\mathbf{8 0 0}$ millions de francs.

\section{Octobre 1991}

1er octobre

Le Conseil des Etats accepte un crédit-cadre de 1"050 millions de francs pour la poursuite de l'aide humanitaire.

\section{8-14 octobre}

Le Conseiller Fédéral René Felber conduit des discussions en Inde sur la conclusion d'un accord de double imposition et de protection des investissements. II visite aussi des projets de développement soutenus 15-17 octobre

par la Suisse.

Assemblée annuelle du FMI et de la Banque Mondiale, Bangkok/ Thaïlande.

15 octobre - 7 novembre 16 octobre

Conférence générale de l'UNESCO, Paris.

Journée mondiale de l'alimentation sur le thème "l'arbre, source de vie", et plusieurs manifestations sur le thème des biotechnologies.

17 octobre

Un Référendum contre l'adhésion de la Suisse au FMI et à la Banque Mondiale est lancé par des organisations de gauche, groupements de 21 octobre femmes et organisations tiers-mondistes.

Le plan mondial de protection de la Terre est lancé dans 65 pays, dont la Suisse, sous la coordination du PNUE, du WWF et de l'Union mondiale de la protection de la nature.

\section{Novembre 1991}

\section{8-12 novembre}

"Congrès mondial des femmes pour une planète saine", organisé par l'organisation féministe Environnement et développement, à Miami, Floride. Les 1.500 participantes adoptent l'Agenda 21 des femmes, un programme d'action pour le 21e siècle (biotechnologies, sciences et 9-27 novembre techniques, environnement, commerce et endettement international). 10 novembre

26ème Conférence générale de la FAO, Rome.

Le Conseil national accepte le nouveau crédit-cadre pour la poursuite de l'aide humanitaire d'un montant de 1"050 millions de francs pour 4 ans. 
18-22 novembre

23 novembre

4ème Conférence générale de l'ONUDI, Organisation des Nations Unies

pour le développement industriel, Vienne.

Forum romand "vaincre la faim". Conférences et débats sur le thème:

Vers un développement équitable et durable.

\section{Décembre 1991}

7 décembre

Réunion nationale du Mouvement pourune Suisse ouverte, démocratique et solidaire, sur le thème du racisme

9-13 décembre

Session extraordinaire du Conseil international du cacao, Londres. 12 décembre

Le Conseil national approuve sans opposition le deuxième crédit-cadre 16-20 décembre

pour l'aide aux pays de l'Est d'un montant de 800 millions de francs.

Conférence des ONG Environnement et développement, en vue de la Conférence de Rio de juin 1992, Paris

\section{Janvier 1992}

26-31 janvier

Une Conférence sur l'eau se tient à Dublin/Irlande, avec la participation de 156 pays, dont la Suisse, et plus de 50 organisations internationales.

Elle attire l'attention sur l'utilisation abusive des réserves d'eau douce et sur la pollution.

27 janvier- 5 février

Afrique, Asie, Amérique latine, 6ème édition du Festival international de films de Fribourg 1992 (repris dans plusieurs villes romandes jusqu'au 29 mars)

28 janvier

Le Conseil des Etats accepte à l'unanimité le 2ème crédit-cadre pour l'aide aux pays de l'Est d'un montant de 800 millions de francs.

\section{Février 1992}

\section{8-25 février}

VIllème Session de la Conférence des Nations Unies sur le commerce 19-21 février

et le développement, Cartagenas de Indias, Colombie.

Session extraordinaire du Conseil international du cacao. 
25-26 février

Sommet mondial en faveur de la promotion économique des femmes en milieu rural, organisé par le Fonds international pour le développement agricole (FIDA) à Genève. Les participantes adoptent une Déclaration de Genève pour les femmes rurales, pour promouvoir une pleine reconnaissance des droits politiques, économiques et sociaux pour les 500 millions de femmes qui travaillent dans le secteur agricole, ainsi que le droit à un salaire égal pour un travail égal.

\section{Mars 1992}

2-13 mars 11-20 mars

8ème Conférence inter. sur les espèces menacées, Kyoto/Japon.

La Suisse participe avec le statut d'observateur à la 36ème Session de la Commission des Nations Unies sur la situation de la femme, à Vienne, pour préparer la Conférence mondiale des femmes qui se tiendra en Chine en 1995.

16-20 mars

CNUCED, Conférence des Nations Unies sur le sucre, Genève. La Conférence adopte un nouvel Accord international sur le sucre, pour remplacer celui qui vient à échéance fin 1992.

\section{Avril 1992}

6-9 avril

6-10 avril

Rencontres Médias Nord-Sud à Genève et Sion, avec des conférences, tables rondes et un concours de films TV sur le développement.

21 avril - 1er mai

CNUCED, deuxième partie de la 38 ème session du Conseil du commerce et du développement, Genève.

21 avril - 1er mai

Session du Conseil international sur le café, Londres

CNUCED, 1ère partie de la Conférence des Nations Unies sur le cacao, Genève. La Conférence entame la négociation en vue d'un nouvel accord international.

22-28 avril

Conférence préparatoire pour la Conférence de Rio de juin 1992.50 pays en développement et 7 pays industrialisés prépare un programme commun à Kuala Lumpur, Malaisie.

24 avril - 3 mai

Campagne d'information en Suisse sur la prostitution des enfants. La campagne met l'accent sur les liens entre l'exploitation sexuelle des enfants dans les pays en développement et le tourisme. 


\section{Mal 1992}

4-14 mai

5 mai

5-18 mai

OMS, 45ème Assemblée mondiale de la santé, Genève.

La Convention de Bâle sur le transport des déchets toxiques entre en vigueur, suite à la ratification de 20 pays, dont la Suisse.

Worlddidac 92 à Bâle, avec une exposition du forum "Ecole pour un monde" sur les thèmes de la libéralisation du commerce, environnement et développement, jeunesse et racisme.

12 mai

Environnement et développement dans les régions de montagne, Rio 92. Des paysans de 4 continents se rencontrent dans le Pays-d'Enhaut, Château-d'Oex.

17 mai

Votation sur l'adhésion de la Suisse au FMI et à la Banque Mondiale. Le peuple suisse accepte cette adhésion.

18-22 mai

CNUCED, 19ème session du Comité spécial des préférences, Genève. 18-30 mai

Premier Congrès mondial sur les peuples indigènes à Rio, Brésil, dans le cadre de lapréparation de la Conférence de Rio. Les peuples indigènes 24-29 mai plaident pour l'autodétermination politique et le droit à un espace vital.

Symposium “Forest 92" sur la recherche sur les forêts tropicales, Rio de Janeiro.

\section{Juin 1992}

1-12 juin

3-24 Juin

Sommet mondial de la terre, Conférence des Nations Unies sur l'environnement et le développement, Rio de Janeiro, Brésil. C'est la plus grande conférence internationale, avec la participation de 172 pays et plus de 5'000 organisations non gouvernementales.

22-26 juin

OIT, 79ème session de la Conférence internationale du travail, Genève.

Session du Conseil international du café à Londres.

Juillet 1992

4-5 juillet

Sommet des 7 pays les plus industrialisés (G7) et sommet économique alternatif à Munich, Allemagne. 
6 juillet

Le Conseil fédéral demande dans son Message au Parlement 600 millions de francs complémentaires pour la coopération avec les pays de l'Est, portant ainsi le Crédit-cadre à 1'400 millions de francs.

6-24 juillet

CNUCED, 2ème session de la Conférence des Nations Unies sur le cacao, Genève.

\section{Août 1992}

21 août

L'Action climat est lancée en Suisse par des oeuvres d'entraide et de développement et les mouvements de protection de l'environnement. La campagne demande l'introduction d'une taxe sur l'utilisation de l'énergie et la mise sur pied d'un Fonds de protection du climat pouvant financer des mesures dans les pays en développement.

\section{Septembre 1992}

3 septembre

Conférence annuelle de la DDA sur le thème du développement en Afrique, Berne.

15-18 septembre

Session du Conseil international sur le cacao à Londres.

16 septembre

Ouverture de la procédure de consultation sur la ratification de la

Convention des Nations Unies pour les droits de l'enfant du 20.11.1989. 21-30 septembre

Session du Conseil international du café à Londres.

22-24 septembre

Session annuelle du FMl et de la Banque Mondiale à Washington. La Suisse devient formellement membre des deux organisations.

28 septembre - 14 octobre

CNUCED, première partie de la 39ème session du Conseil du commerce et du développement, Genève.

\section{Octobre 1992}

9 octobre

Vote final du Parlement sur l'arrêté fédéral sur les coupures linéaires des dépenses de la Confédération pour les années 1993 à 1995. Les budgets de la coopération sont coupés d'un demi milliard de francs par rapport au Plan financier. 
16 octobre

Journée mondiale de l'alimentation sur le thème anourriture et alimentation". Diverses actions sont entreprises en Suisse sur les conséquences de la suralimentation au Nord et sur la croissance de la population dans le Sud.

19-25 octobre

Semaine d'information pour une Suisse sans fuite de capitaux, organisée par une coalition d'organisations de développement de milieux d'églises et de syndicats, en lien avec la campagne 500 ans d'oppression - 500 ans de résistance en Amérique latine.

Novembre 1992

2-13 novembre

3ème session de la Conférence des Nations Unies sur le cacao, Genève. 18-19 novembre

Symposium "Agriculture et environnement" à Berne, organisé par

Swissaid, WWF Suisse et la Ligue suisse pour la protection de la nature. 30 novembre 4 décembre

Première conférence de la Convention de Bâle à Montevideo, Uruguay.

Décembre 1992

4 décembre

Le Conseil des Etats accepte le crédit complémentaire sur l'aide aux pays de l'Europe centrale et orientale de 600 millions, portant cette aide à 1,4 milliards de francs.

5-11 décembre

OMS et FAO, Conférence des Nations Unies sur l'alimentation et la 15 décembre santé, Rome.

Les représentants de la Banque Mondiale et de 34 pays donateurs se mettent d'accord à Berne sur la 10ème reconstitution du capital de l'Agence internationale du développement (IDA), d'une valeur de 13 milliards de DTS. 